\title{
A comparison of braking strategies for elastic joint robots
}

\author{
Nico Mansfeld and Sami Haddadin
}

\begin{abstract}
It has recently been shown that intrinsically elastic robots are capable of outperforming rigid robots in terms of peak velocity by making systematic use of energy storage and release. Certainly, high link side velocities are beneficial for performance, however, they also increase the probability of self damage or human injury in case of a collision. To ensure the physical integrity of both human and robot, it is therefore crucial to avoid potentially dangerous collisions and react in a compliant manner if unwanted contact has occurred or may occur unforeseeable. In this paper, we consider the most intuitive collision anticipation and pre-reaction scheme, namely stopping an elastic robot, if possible in minimum time. For 1-DOF elastic joints with limited elastic deflection we extend existing model-based and model-free controllers and compare their performance. Furthermore, we analyze the braking trajectory that is achieved with the different strategies. The 1-DOF solution is extended to the double pendulum case, where we show that feasible estimates for maximum and final position can be obtained at the very first instant of braking.
\end{abstract}

\section{INTRODUCTION}

Many of today's robots are being developed for close interaction with humans in either industrial or domestic environments. One key issue in physical human-robot interaction (pHRI) is safety, because it is primary to ensure that a human is not harmed in any situation. For this, a robot must be designed and controlled such that no potentially dangerous collision occurs. In particular, one is interested in minimizing harm already prior to the collision.

In robotics, there exist many pre-collision strategies to generally avoid contact with the environment [1], [2], [3] or to limit the robot speed to a biomechanically safe value such that human injury can be avoided upon contact [4]. In the context of safe motion control, one important problem is to stop a robot as fast as possible. For rigid robots with stiff position control, there exist standardized schemes for braking according to DIN EN 60204, e.g. engaging the brakes and switching off the drives at the same time (category 0 ) or commanding a stopping trajectory using the maximum available power (category 1 ). Such strategies might also be employed for torque controlled rather rigid designs such as the LWR family, which show compliance via active control.

More recently, it has become increasingly popular to introduce intrinsic elasticity for achieving compliance already in the mechanical structure [5], [6], [7]. Another motivation for introducing deliberate joint elasticity is to exploit the energy storage and release capabilities to outperform rigid robots by means of energy efficiency and peak velocity [8], [9], [10]. Considering safety, however, the benefit of joint elasticity on collision safety has to be treated differentiated. Elasticity may improve compliance on the one hand, but high link side velocities increase human injury probability during contact on the other hand [11], [12]. The problem of reducing injury

Nico Mansfeld is with the Institute of Robotics and Mechatronics, German Aerospace Center (DLR), Wessling, Germany, Sami Haddadin is with the Institute of Automatic Control, Leibniz University Hannover (LUH), Germany, nico.mansfeldedlr.de, sami.haddadin@irt.uni-hannover.de

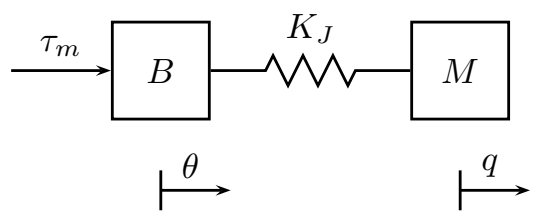

Fig. 1. 1-DOF elastic joint

risk already before a collision occurs is therefore also an important problem for this class of robots.

In this paper, we consider the most basic pre-collision scheme for elastic robots, namely effective braking. In optimal control literature, there exists the time-optimal solution for braking of linear visco-elastic joints [13]. In [14] this problem was extended by taking also limited elastic deflection into account. It was shown that the SISO control law can be applied to multi-DOF robots by using a decoupling based control scheme. An alternative method was presented in [15], which makes use of the energy storage and release properties of elastic joints to develop a passive, model-free braking scheme. The aim of the present work is to extend and compare existing model-based with model-free braking strategies for 1-DOF elastic joints. Furthermore, we analyze the braking trajectory for the respective controllers to predict the braking distance of an elastic robot. A double pendulum is finally used to exemplify the application of braking control and braking distance estimation to $n$-DOF robots.

This paper is organized as follows. In Sec. II we introduce the elastic joint model considered in this work. In Sec. III we formally define the considered control problem, describe existing braking controllers and propose modifications for improving their performance. The case of limited elastic deflection as well as the applicability to nonlinear joints and full $n$-DOF robots are discussed. Braking distance estimation for 1-DOF elastic joints and the extension to a double pendulum are considered in Sec. IV. Finally, Sec. V concludes the paper.

\section{CONSIDERED MODEL}

The dynamics of a single visco-elastic robot joint consisting of motor, elastic transmission, and link (see Fig. 1) are governed by following differential equations

$$
\begin{aligned}
B \ddot{\theta} & =\tau_{m}-\tau_{f}-\tau_{J} \\
M \ddot{q} & =\tau_{g}-\tau_{J} \\
\tau_{J} & =K_{J}(\theta-q),
\end{aligned}
$$

where the motor inertia and position are denoted $B$ and $\theta$, the constant joint stiffness $K_{J}$, and the link inertia and position $M$ and $q$. The motor and motor friction torque are $\tau_{m}$ and $\tau_{f}$, the elastic joint torque is $\tau_{J}$. For the principle analysis we consider in this paper, we make following assumptions. 
First, we assume that gravity torques are being compensated. Furthermore, we assume $\tau_{f} \approx 0$ for sake of clarity.

As the intention of this paper is to unveil basic principles of intrinsically elastic joints, we take the most important realworld constraints of such devices into account, namely the maximum motor velocity and elastic deflection

$$
\begin{aligned}
& |\varphi|=|\theta-q| \leq \varphi_{\max } \\
& |\dot{\theta}| \leq \dot{\theta}_{\max } .
\end{aligned}
$$

Typically, the motor dynamics of intrinsically elastic joints are significantly faster than the link side dynamics. In this work, we thus model the motors as velocity sources, meaning desired velocities complying with (5) can be reached instantaneously under all operating conditions. The validity of this singular perturbation approach was already experimentally shown in [8], [15]. The reduced dynamics can now be expressed as

$$
\begin{aligned}
& \theta=\int \dot{\theta} d t+\theta_{0} \\
& \ddot{q}=\omega^{2}(\theta-q),
\end{aligned}
$$

where $\theta_{0}$ is the initial motor position, $\omega=\sqrt{K_{J} / M}$ the mass-spring eigenfrequency, and $T_{p}=2 \pi / \omega$ the periodic time. We select the motor velocity as the control input $u:=$ $\dot{\theta}$, and the elastic deflection and link velocity as the system state $\boldsymbol{x}:=[\dot{q} \varphi]^{T}$. Now, the first order differential equations are found to be

$$
\dot{\boldsymbol{x}}=A \boldsymbol{x}+B u=\left(\begin{array}{cc}
0 & \omega^{2} \\
-1 & 0
\end{array}\right) \boldsymbol{x}+\left(\begin{array}{l}
0 \\
1
\end{array}\right) u .
$$

For the examples shown in this paper, we select the system parameters $M=1.8 \mathrm{~kg}, K_{J}=207 \mathrm{Nm} / \mathrm{rad}, u_{\max }=2 \mathrm{rad} / \mathrm{s}$, and $\varphi_{\max }=0.1 \mathrm{rad}$ if boundary control is considered.

\section{1-DOF BRAKING CONTROLLERS}

In this section, we discuss five different methods to brake an elastic joint. Two of them take advantage of the full joint dynamics, while the other three assume unknown system parameters. First, we provide a formal definition of the problem. Then, we analyze the energetic behavior of the elastic joint and how the equilibrium can be reached. This influences the design of each controller described hereafter.

\section{A. Problem formulation}

Essentially, we seek to stop the elastic joint as fast as possible, i.e. reach the equilibrium $\left[\begin{array}{ll}0 & 0\end{array}\right]^{T}$ without violating (4) and (5). Note that we are explicitly interested in extracting energy from the system and not in stopping at a desired goal state. The initial and final conditions for this problem are

$$
\begin{array}{cc}
\dot{q}(0)=\dot{q}_{0}, & \dot{q}\left(t_{f}\right)=0 \\
\varphi(0)=\varphi_{0}, & \varphi\left(t_{f}\right)=0,
\end{array}
$$

where $t_{f}$ is the final time and $\boldsymbol{x}_{0}:=\left[\begin{array}{ll}\dot{q}_{0} & \varphi_{0}\end{array}\right]^{T}$ the initial state. In order to meet the constraints while braking, this state must be located within a brakable set $\mathcal{B}$. In [14], this set was determined for time-optimal braking. The respective analysis may be used to find the brakable set also for the other controllers described in this work. However, this is goes beyond the scope of the present paper. Here, we assume that the mass-spring system is always brakable, i.e. $\boldsymbol{x}_{0} \in \mathcal{B}$.

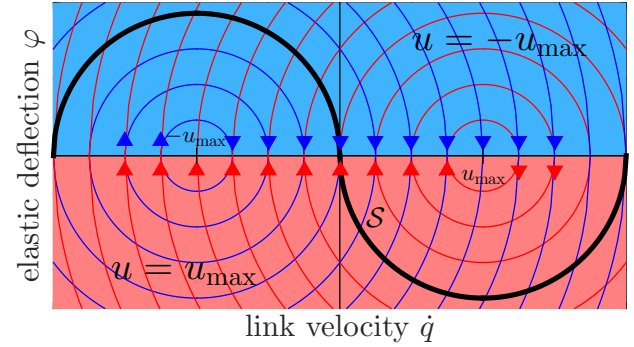

Fig. 2. System behavior if control law (14) is used. The state travels clockwise on the blue ellipses if the minimum motor speed is applied and on the red ellipses if the maximum velocity is applied.

\section{B. Remark: Reaching equilibrium}

The overall system energy of the considered elastic joint is

$$
V=U+T=\frac{1}{2} K_{J} \varphi^{2}+\frac{1}{2} M \dot{q}^{2},
$$

where $U$ is the spring potential energy and $T$ the link kinetic energy. The change of energy is simply found to be

$$
\dot{V}=K_{J} \varphi u \text {. }
$$

A very intuitive energy dissipating idea would thus be to choose the control law

$$
u=-K_{c} \varphi
$$

based on (12), where $K_{c}$ is the controller gain. We seek for maximum controller performance, i.e. the motor travels at maximum/minimum velocity ${ }^{1}$. The control law (13) then becomes

$$
u=-\operatorname{sign}(\varphi) u_{\max } .
$$

This control law reduces the system energy locally. However, the trajectory may not converge to the equilibrium, but remains at a point on the line segment $\dot{q} \in\left[-u_{\max }, u_{\max }\right]$, $\varphi=0$. This is because the ellipses centered around $\pm u_{\max }$ meet on this line segment with opposite direction of travel, see Fig. 2 Because $\varphi=0$, no elastic torque is available to brake the link to zero velocity. In this situation, one may switch to a different controller to reach the equilibrium. This can be e.g. one of the other controllers described in this work. For reaching the equilibrium directly, it can be observed from Fig. 2 that the system state must travel on the curve denoted $\mathcal{S}$.

Next, the first model-based controller is described. For clarity of presentation, we first consider the unbounded case, meaning the state constraint (4) is not active. Boundary control is considered after describing every model-based and model-free controller.

\section{Time-optimal control}

The time-optimal solution for braking an elastic joint with dynamics (7) is a standard problem in optimal control theory [13]. The switching curve $\mathcal{S}$ can be obtained when starting at the equilibrium and applying $u= \pm u_{\max }$ for one half periodic time $T / 2=\pi / \omega$, resulting in one half ellipse, see Fig. 3 (upper left). The adjacent half ellipses are defined by starting a new half ellipse from every point of the first

\footnotetext{
${ }^{1}$ Please note that this choice does not alter the principle behavior of any controller described in this paper.
} 

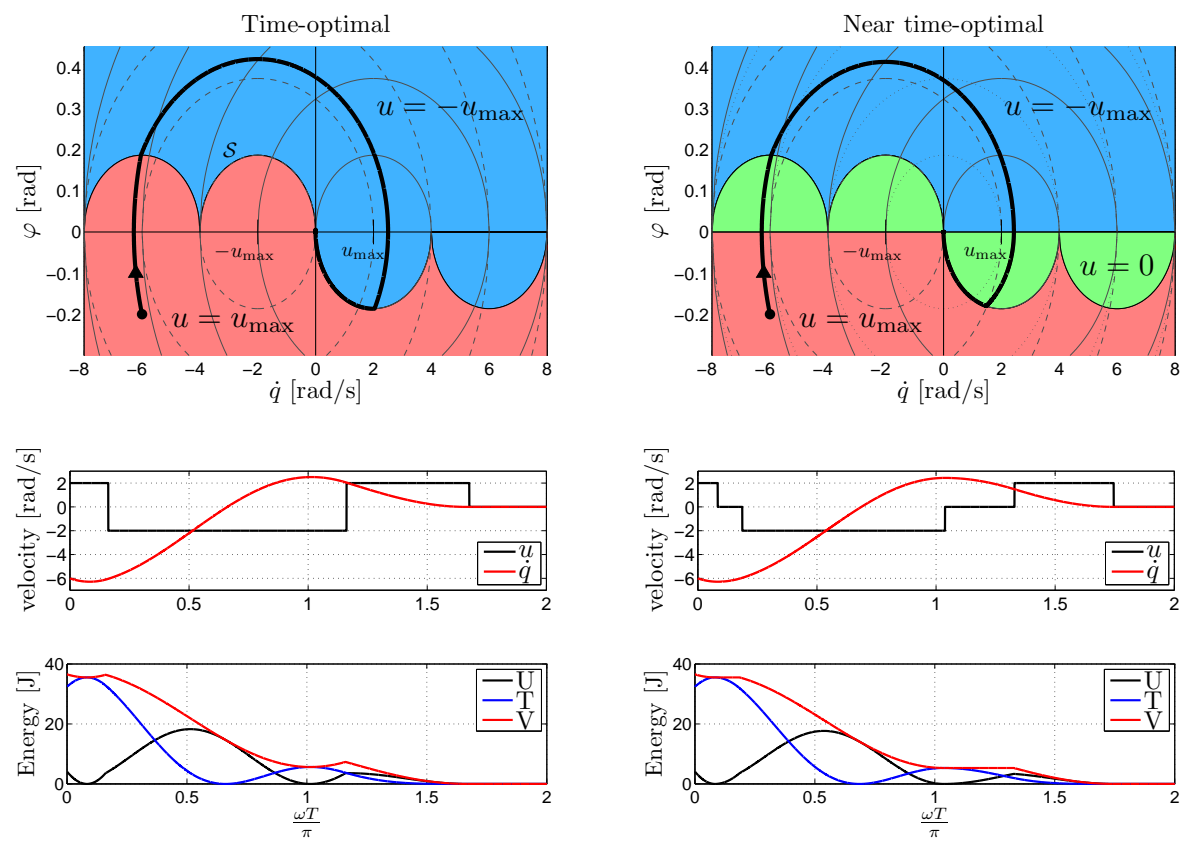

Fig. 3. Time-optimal (left column) and near-time optimal (right column) control law. In the upper row, the phase plane representation of the control laws as well as an exemplary trajectory are depicted. The gray solid, dashed, and dotted lines represent ellipses centered around $u_{\mathrm{max}},-u_{\mathrm{max}}$, and the origin, respectively. The motor and link velocities are depicted in the middle row, the system energies in the lower row. The $x$-axis indicates the time $\frac{\omega T}{\pi}$ in half-cycles of the system eigenfrequency.

ellipse, which physically means eigenfrequency excitation. Successively repeating this procedure provides the remaining ellipses of the switching curve. In order to hit zero velocity and deflection time-optimally, one must apply $u=u_{\max }$ if the current state is located below $\mathcal{S}_{r}$, above the switching curve $u=-u_{\max }$ has to be commanded. The control law can be formulated as

$$
\begin{aligned}
i_{\mathcal{S}} & =\left\lceil\frac{|\dot{q}|}{2 u_{\max }}\right\rceil \\
\varphi_{i_{\mathcal{S}}} & =-\frac{\operatorname{sign}(\dot{q})}{\omega} \sqrt{u_{\max }^{2}-\left(|\dot{q}|-\left(2 i_{\mathcal{S}}-1\right) u_{\max }\right)^{2}} \\
u_{T O C} & =\operatorname{sign}\left(\varphi_{\mathcal{S}}-\varphi\right) u_{\max }
\end{aligned}
$$

where $i_{\mathcal{S}}$ is the index of the switching curve located below/above the system state and $\varphi_{i_{\mathcal{S}}}$ the deflection of the switching curve with same velocity $\dot{q}$ as the link.

The control law is depicted in Fig. 3 (upper left) where the red area represents maximum velocity input and the blue area minimum velocity input. In the phase plane, the black solid line represents an exemplary braking trajectory. The according motor and link velocities are shown in the middle figure. In the bottom figure, the spring potential energy $U$, link kinetic energy $T$, and total system energy $V=U+T$ are depicted. The overall system energy is being reduced as fast as possible. However, it is not decreasing monotonically, c.f. Fig. 3 (lower left) at approx. 0.2 and 1.2 half cycles. In the first and third quadrant in the phase plane, energy decrease, i.e. $\dot{V} \leq 0$ according to (12), is always ensured. In the second and fourth quadrant, however, the system energy increases when the current state is located within the area enclosed by the switching curve and the $x$-axis. This is because motor velocity and elastic deflection have equal sign. This results in a non-passive behavior.
In control, passivity is generally a desirable property due to being strongly related to stability and robustness. Next, we propose a modification of the time-optimal control law, which leads to suboptimality by means of braking time but monotonic energy decrease, i.e. the controller shows passive behavior.

\section{Near time-optimal \& passive control}

To achieve passivity, we cannot select positive motor velocity in the left half of the area enclosed by the first switching curve, the $x$-axis and $u_{\max }$, because the system may hit and remain at a point on the line segment $\dot{q} \in\left[-u_{\max }, u_{\max }\right]$, $\varphi=0$ as described in Sec. III-B and illustrated in Fig. 2. Thus, we choose $u=0$ in this area. When applying zero velocity, the energy of the system remains constant and energetically passive behavior is achieved. In the phase plane, the system state travels on an ellipse centered around the origin until the switching curve $\mathcal{S}$ is hit, see Fig. 3 (upper right). The maximum motor velocity can then be commanded because deflection and motor velocity have different signs, which in turn results in overall energy decrease. For the right half of the area enclosed by the $x$-axis, $\mathcal{S}$ and $u_{\max }$, we could apply the control law (14) to reduce the system energy. However, then we must apply $u=u_{\max }$ after entering this area, switch to $u=0$ if $\dot{q}<u_{\max }$ and again command $u=u_{\max }$ after hitting the switching curve. This does not only require many motor switchings, but may also result in a long braking time. Therefore, we choose $u=0$ in this area. We also select zero motor velocity for the remaining areas enclosed by the switching curve, because then we can use the switching curves of the time-optimal control law and only apply slight modifications to achieve passivity. In summary, 

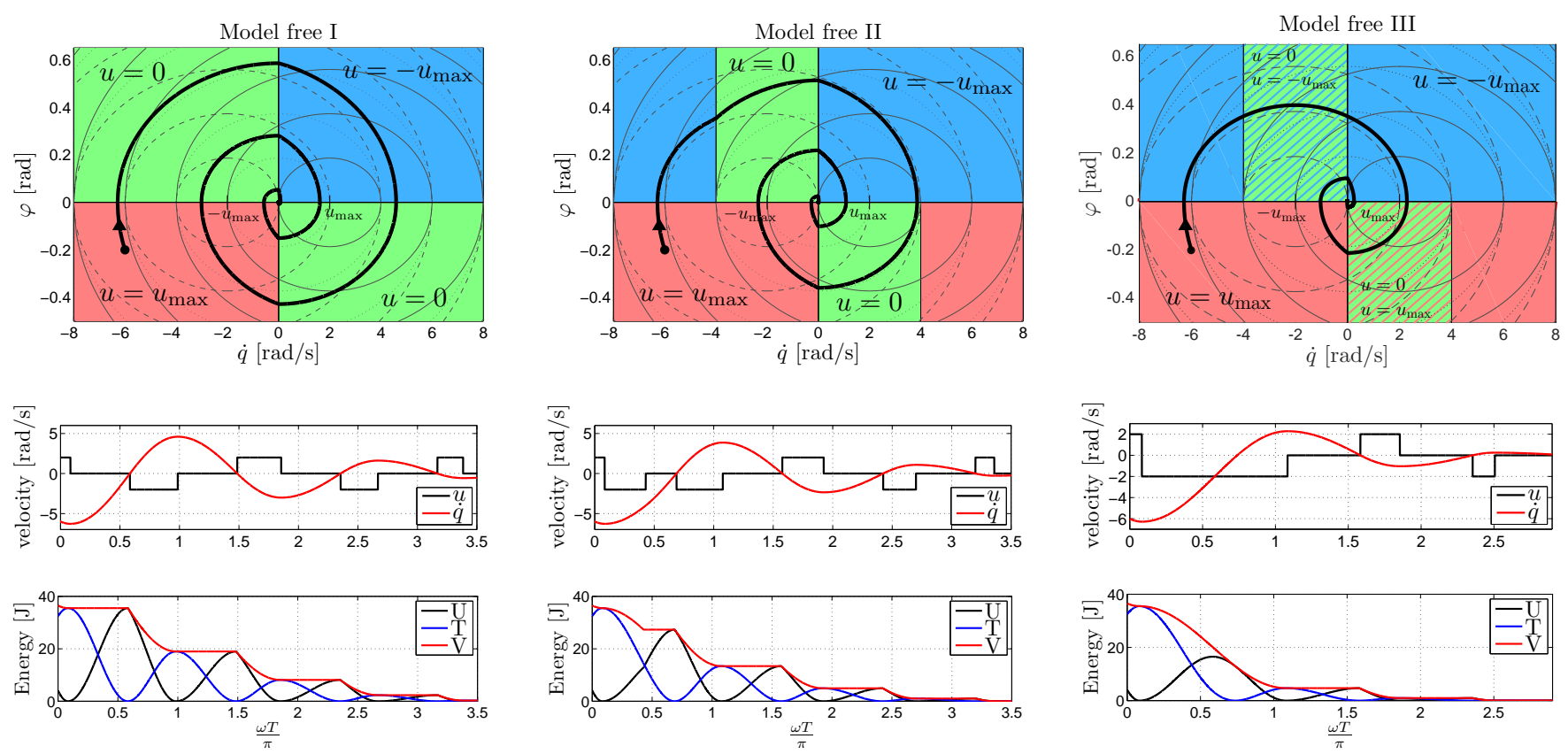

Fig. 4. Model-free control law I, II, and III with exemplary braking trajectories.

the control law for near time-optimal control is given by

$$
u_{\text {NTOC }}= \begin{cases}-\operatorname{sign}(\dot{q}) u_{\max }, & \varphi \dot{q} \geq 0 \\ \operatorname{sign}(\dot{q}) u_{\max }, & \varphi \dot{q}<0 \wedge|\varphi| \geq\left|\varphi_{i, \mathcal{S}}\right| \\ 0, & \text { otherwise }\end{cases}
$$

The phase plane representation together with an exemplary trajectory is depicted in Fig. 3 (upper right), where the green areas represent zero velocity and the blue and red area minimum and maximum motor velocity, respectively. From the timely evolution of the total system energy depicted in Fig. 3 (lower right) it can be observed that passivity and only slightly larger braking time in comparison to time-optimal control are achieved.

The previous two control laws require full knowledge of the dynamics. Next, we consider the case of unknown system parameters, i.e. link inertia and joint stiffness. We only assume that the maximum and minimum values of $u=\dot{\theta}$ are known.

\section{E. Model free control I}

The switching curves for time-optimal or near timeoptimal control depend on the system eigenfrequency and motor velocity. Because the eigenfrequency is assumed to be unknown, we cannot derive a switching curve to decide on the control input in quadrant two and four. In quadrants one and three, however, there are no switching curves in the previous two methods. Therefore, the control law for both model-based and model-free controllers may be the same in these phase plane areas. To take this into account, following control law was proposed in [15].

$$
u_{M F I}= \begin{cases}-\operatorname{sign}(\dot{q}) u_{\max }, & \varphi \dot{q} \geq 0 \\ 0, & \text { otherwise }\end{cases}
$$

In quadrants two and four, the motor velocity is set to zero, i.e. the energy remains constant. This leads to an overall energetically passive behavior of the controlled oscillator. The phase plane representation of the control law is illustrated in Fig. 4 (upper left), the system energy for an exemplary initial state in Fig. 4 (lower left).

It is important to notice that the equilibrium can only be reached asymptotically with this control law, in other words the joint cannot be stopped in finite time without frictional or damping effects if the system trajectory does not coincide with the first switching curve. In the controller presented next, we modify this model-free approach to achieve faster energy decrease.

\section{F. Model free control II}

As mentioned in Sec.III-B and Sec. III-D we want to avoid applying maximum/minimum motor speed in the area enclosed by the first switching curve and the $x$-axis. Generally, we have no information about the maximum deflection of the switching curve because the mass-spring eigenfrequency is unknown. However, we know that its maximum width is $2 u_{\text {max }}$, see e.g. Fig. 3 (upper left). Therefore, we can apply maximum velocity and achieve energy decrease for $|\dot{q}| \geq 2 u_{\max }$. For smaller link velocities, we then choose $u=0$. In summary, the control law can be written as

$$
u_{M F I I}= \begin{cases}-\operatorname{sign}(\dot{q}) u_{\max }, & \varphi \dot{q} \geq 0 \\ \operatorname{sign}(\dot{q}) u_{\max }, & \varphi \dot{q}<0 \wedge|\dot{q}| \geq 2 u_{\max } \\ 0, & \text { otherwise }\end{cases}
$$

The according phase plane representation is depicted in Fig. 4 (upper middle). From the timely evolution of energy it can be observed that the energy decreases monotonically and faster than for the previous controller. However, if the initial velocity is $|\dot{q}|<2 u_{\text {max }}$ then the control law is identical with 


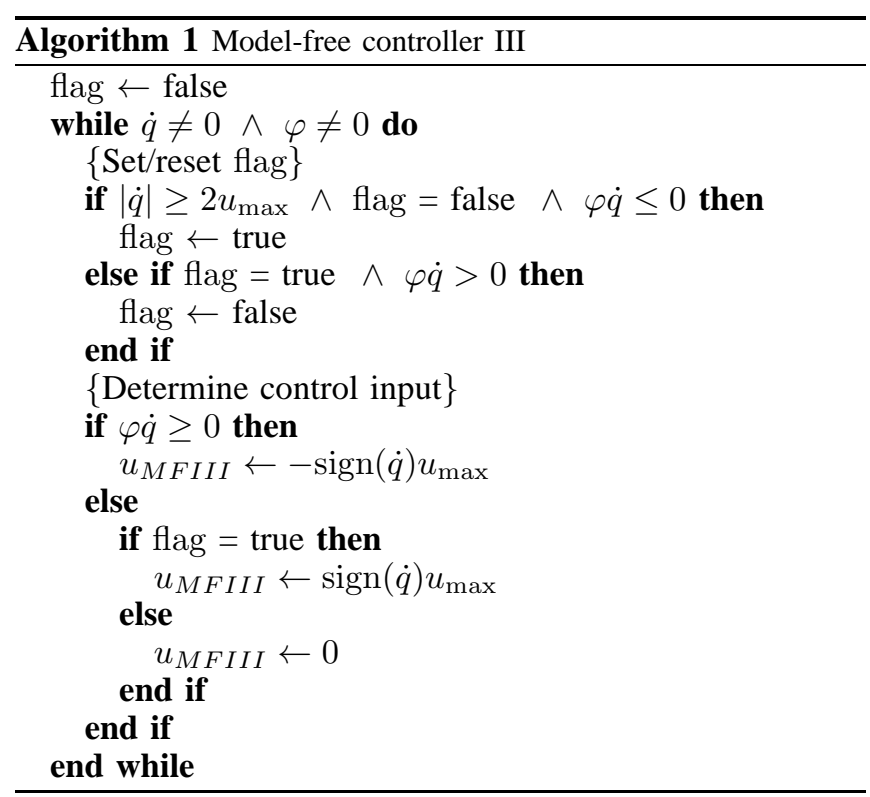

the previous one. In the following, a further modification for improving braking performance is presented.

\section{G. Model free control III}

If the initial link velocity is $|\dot{q}| \geq 2 u_{\max }$ then the system travels on an ellipse which has a larger distance to $\pm u_{\max }$ than the first switching curve if maximum/minimum motor speed is commanded. In this case, one can continue traveling with $\pm u_{\max }$ even if $|\dot{q}|<2 u_{\max }$ and achieve faster energy decrease. For the according control law, one has to remember/save the the information about the initial velocity when braking is initiated or a new quadrant is being entered in the phase plane. In the sequel, this variable is denoted flag. The control law is listed in algorithm Alg. 1, an example in the phase plane is depicted in Fig. 4 (upper right).

In the example, we set flag to 1 when entering quadrant two because $\dot{q}<-2 u_{\max }$ and apply $u=-u_{\max }$ until we reach the first quadrant. When hitting the first quadrant flag is being reset. The fourth quadrant is being entered at $\dot{q}<$ $2 u_{\max }$ which means that flag remains inactive and $u=0$ has to be applied.

Another possible extension for the presented controllers is to command $u=\dot{q}$ if $|\dot{q}| \leq u_{\max }$ in quadrant two and four. If the motor travels with the same velocity as the link, then the deflection, in other words potential energy, remains the same while the kinetic energy is being reduced until $u=\dot{q}=0$. If enough elastic torque torque is available, then this control can lead to faster energy decrease than only choosing $u=0$ in quadrant two and four. However, the discussion of this extension is subject to future work.

\section{H. Boundary control}

In [14] the set of brakable states and the according timeoptimal trajectories were found for time-optimal braking of joints with limited elastic deflection. Quasi-singular arcs may occur if the maximum elastic joint deflection is lower than the maximum deflection $\varphi_{\mathcal{S}, \max }=\frac{u}{\omega}$ of the first switching curve. When hitting the constraint, one must apply $u=\dot{q}$ and follow the maximum deflection until the switching curve is reached. After hitting the switching curve, maximum motor

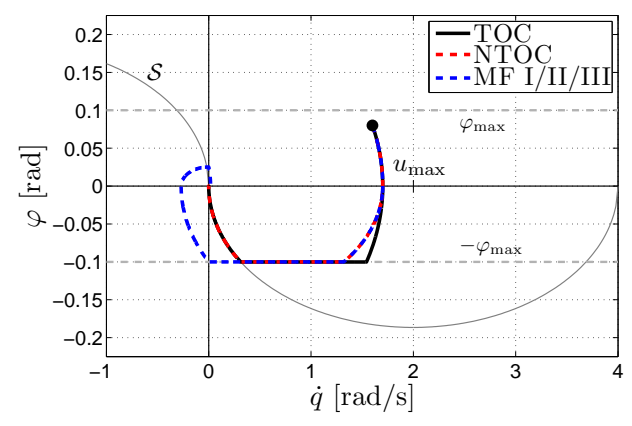

Fig. 5. Boundary control in case the maximum joint deflection $\varphi_{\max }$ intersects the switching curve $\mathcal{S}$. An exemplary braking trajectory is depicted for time-optimal (TOC), near time-optimal (NTOC) and model free (MF I/II/III) control. The according motor velocities and timely evolution of energies are depicted in Fig. 6.
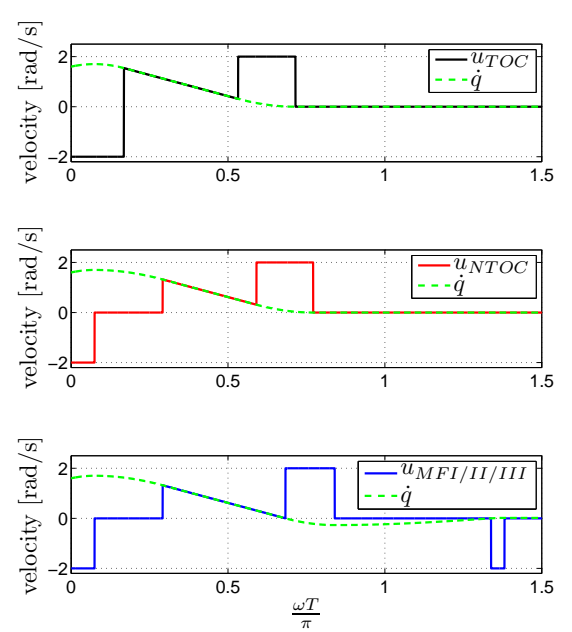

Fig. 6. Motor and link velocities for the quasi-singular braking trajectories shown in Fig. 5. Time-optimal control is depicted in the upper, the near time-optimal in the middle, and the model free control in the lower figure.

velocity has to be commanded to reach the equilibrium. This is exemplified in the phase plane trajectory from Fig. 5 and the respective motor velocity in Fig. 6 (upper). Boundary control is only possible if the system reaches the constraint at $|\dot{q}| \leq u_{\max }$. Otherwise it is not brakable without violating the maximum elastic deflection.

For the near time-optimal control law, we select the same boundary control. The deviation from the time-optimal law is that zero velocity is applied in the area enclosed by the first switching curve, which results in passivity but in longer braking time, see Fig. 6 (middle). In case the maximum elastic deflection is known by the model-free controllers, one can set $u=\dot{q}$ as long as $\dot{q} \geq 0$. This ensures no violation of the deflection constraint, see Fig. 5. Of course, if the deflection constraint is unknown, then it may eventually be exceeded.

\section{Controller comparison and discussion}

In this section, we compare the controller performance and comment on the applicability to nonlinear stiffness and bounded motor dynamics, and the extensibility to $n$-DOF elastic robots. Since the aim of this paper is to extend existing model-free and model-based braking controllers and describe their basic properties, the experimental verification is subject 

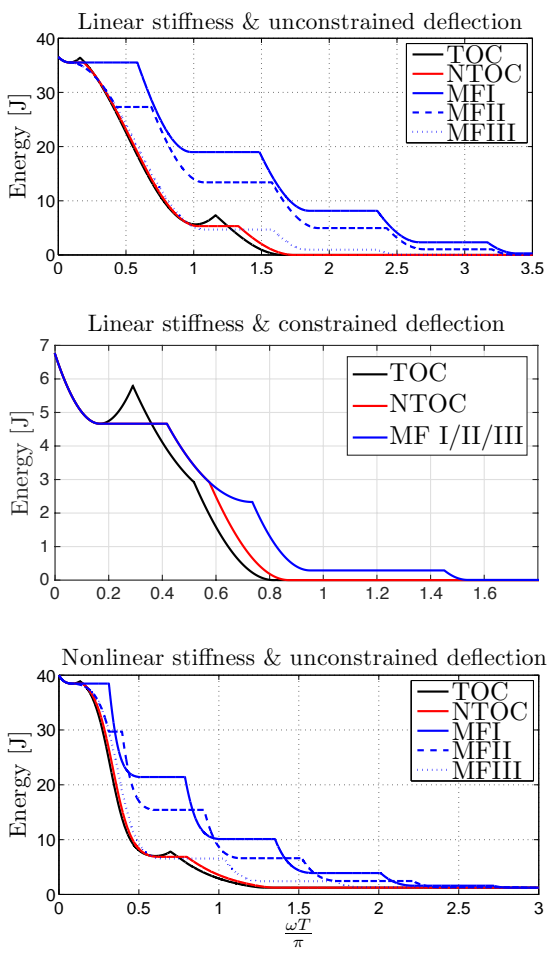

Fig. 7. Timely evolution of system energy $V$ for all controllers in case of linear stiffness and unconstrained elastic deflection (upper), linear stiffness and boundary control (middle), and nonlinear stiffness and unconstrained deflection (lower)

to future work.

a) Performance: For comparing the performance of the different algorithms, we start with the same initial conditions for all cases. In Fig. 7 (upper) the total system energy over time is illustrated for the unconstrained, linear case and in Fig. 6 (middle) for the constrained, linear case. The timeoptimal solution is of course the fastest, however, the energy does not decrease monotonically. The near-time optimal control law ensures passivity and is only slightly slower than the time-optimal scheme. The methods without parameter knowledge are passive, but show inferior performance and only asymptotic convergence. For the selected initial conditions the schemes proposed in this work show better performance than the one described in [15]. However, as already explained, the model-free approaches are identical in case of boundary control (constrained case), and if $|\dot{q}| \leq 2 u_{\text {max }}$. Among the model-free controllers, no. III performs best. The only drawback comes rather from the implementation side. By storing the information about the initial velocity, it is assumed that the trajectory travels on a certain ellipse outside the switching curve in the phase plane. If external torques act on the joint, e.g. by a collision or coupling effects from other joints in multi-DOF robots, then the system state can be brought to the area enclosed by the first switching curve, leading to an undesired motor input.

b) Nonlinear elasticity: The model-based controllers described in this work were developed for joints with linear elasticity. If nonlinear joint stiffness is present, these controllers will clearly not perform as good as the optimal solution, which has to be found by solving a new optimal control problem. However, we may now analyze whether the two model-based controllers provide suboptimal, however, feasible motor velocities if the system dynamics are being linearized along the trajectory. For this we replace the linear spring by the progressive, nonlinear torque/deflection relationship of a FSJ mechanism [16]. The elastic torque for this device is defined as

$$
\tau_{J, F S J}(\varphi)=68.7\left(e^{12.5\left(\varphi-\varphi_{\max }(\sigma)\right)}-e^{12.5\left(-\varphi-\varphi_{\max }(\sigma)\right)}\right),
$$

where we use $\varphi_{\max }(\sigma)=10 \mathrm{rad}$. Alternatively, this could be adjusted by a second motor. For sake of brevity we do not take deflection constraints into account. For comparing the controller performance, we select the same initial conditions as in the unconstrained, linear case. The results are depicted in Fig. 7 (lower). In this example, one can observe that all model-based and model-free controllers show the same principle behavior regarding braking time and passivity as in case of linear stiffness. A full analysis and experimental verification are still to be done in future research.

c) Limited motor dynamics: For analyzing the influence of limited motor acceleration on the braking performance, we assume that the motor has the PT1 dynamics

$$
u^{\prime}=\frac{1}{T_{m} s+1} u,
$$

where $T_{m}$ is the time constant and $u^{\prime}$ the new motor speed. Furthermore, we add white noise with $1 \mathrm{kHz}$ frequency and $10^{-6} \mathrm{~W} / \mathrm{Hz}$ power spectral density to the link velocity, which is e.g. larger than the noise in the DLR Hand Arm System [7]. To determine the influence of limited motor acceleration on the final braking time we choose the $95 \%$ rise time of the motor speed to be $5,10,15,20$, and $25 \%$ of the half periodic time of the mass-spring system, i.e. approx. $T_{m}=\frac{3 x}{100} \frac{\pi}{\omega}, x=\{5,10,15,20,25\}$. For linear joint stiffness, unconstrained elastic deflection, and $25 \%$ motor acceleration time the timely evolution of total system energy $V$ is depicted in Fig. 8 (upper). It can be observed that the model-free controllers and the near time-optimal controller are not strictly passive anymore. This is because the motor velocity and elastic deflection have the same sign in quadrant two and four until the motor reaches zero velocity, c.f. Fig 4. This leads to $\dot{V}>0$ according to (12). Due to the bangbang structure of the time-optimal control law, oscillations now remain at the end of the braking motion. For avoiding these, a different controller such as a well-tuned position controller could be activated if most of the system energy was removed as well as the elastic deflection $\varphi$ and link velocity $\dot{q}$ go below a certain threshold $\varphi_{\epsilon}$ and $\dot{q}_{\epsilon}$.

The influence of motor acceleration capability on the final braking time is depicted in Fig. 8 (lower). Here, we define the final braking time as the time that is required until $95 \%$ of the initial energy was removed from the system. It can be observed that increasing $T_{m}$ generally results in larger braking time. While for the model-based controllers the difference is only moderate, it is significant for the modelfree controllers, because the system trajectory has to pass quadrant two or four with zero energy decrease one more time.

d) Extension to $n$-DOF: Up to now, the described controllers were suited for 1-DOF elastic joints only. For braking a multi-DOF robot, intuition tells that simultaneous braking of every joint results in a stopping motion of the 

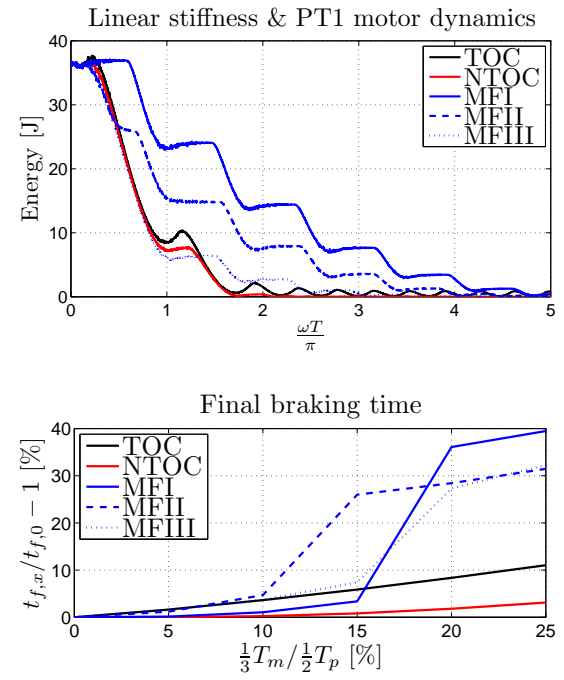

Fig. 8. In the upper figure, the timely evolution of system energy $V$ is depicted for a mass-spring system with linear stiffness, unbounded elastic deflection, PT1 motor dynamics, and noise in the link velocity. In the lower figure, the influence of limited motor dynamics on the final braking time is illustrated. The $x$-axis indicates the ratio of motor acceleration time and one half the periodic time $0.5 T_{p}$ of the oscillator, the $y$-axis the ratio of the braking time $t_{f, x}$ for limited motor dynamics and the braking time $t_{f, 0}$ for unlimited motor acceleration.

entire robot $^{2}$. For achieving this, the 1-DOF controllers may be implemented in the multi-DOF a) joint space, or b) decoupled space.

In [14] we proposed a decoupling-based control scheme which decouples both the dynamics and control region, i.e. the motor velocities, for enabling SISO control in modal coordinates. Because the approach is model-based, it is possible to implement the time-optimal or near time-optimal control law, which will most likely show a better performance than the model-free control laws.

If control in joint coordinates is considered, then the model-free controllers I and II can easily be implemented while care has to be taken for the third controller (see above). For the model-based methods, one has to linearize the dynamics and approximate the "eigenfrequency" of the respective joint required to describe the switching curve, because the real system eigenfrequencies obtained by eigenvalue computation cannot be assigned to a particular joint. However, the performance and stability analysis for $n$-DOF robots goes beyond the scope of this paper and is subject to future research.

\section{BRAKING DISTANCE ESTIMATION}

Up to now we were interested in how fast we can decrease the system energy to achieve effective braking. For avoiding unwanted contacts with obstacles or humans, it is furthermore beneficial to know at which position the robot will stop and even more which trajectory it will take until standstill. A priori knowledge of the braking distance may then be used to initiate braking such that no possibly hazardous collision occurs.

Let us consider the final braking time $\hat{t}_{f, i}$ as well as the link trajectory $\hat{q}_{i}(t), t \in\left[t_{0}, \hat{t}_{f, i}\right]$ of each joint with index $i \in\{1, \ldots, n\}$ can be predicted at start of braking,

\footnotetext{
${ }^{2}$ Certainly, proof has to be found in future work.
}

denoted $t_{0}$. For a full $n$-DOF robotic manipulator, it is then possible to make use of the forward kinematics to combine the state estimation of each joint. One may predict the final Cartesian position $\hat{\boldsymbol{x}}_{P O I, t_{f}} \in \mathcal{R}^{3}$ and orientation $\hat{R}_{P O I, t_{f}} \in \mathcal{R}^{3 \times 3}$ of a particular point of interest (POI) along the robot structure as well as the according braking trajectory $\hat{\boldsymbol{x}}_{P O I}(t)$ and $\hat{R}_{P O I}(t)$, where $t \in\left[t_{0}, \hat{t}_{f}\right]$. Here, the estimated final time of the entire robot is the worst case braking time of all joints, i.e. $\hat{t}_{f}=\max \left(\hat{t}_{f, i}\right), i \in$ $\{1, \ldots, n\}$. Let an obstacle in the environment be represented by a set of Cartesian points denoted $\mathcal{X}_{\text {obs }}$. The distance of a POI on the robot structure with position $\boldsymbol{x}_{P O I}$ to the obstacle $^{3}$ can be determined by $d=\min \_\operatorname{dist}\left(\boldsymbol{x}_{P O I}, \mathcal{X}_{\mathrm{obs}}\right)$, where the function min_dist can e.g. be realized with the GJK algorithm [17]. Given the full braking trajectory, it is possible to estimate the smallest distance during stopping, namely $\hat{d}_{\text {min }}=\min \left(\min \_\operatorname{dist}\left(\boldsymbol{x}_{P O I}(t), \mathcal{X}_{\text {obs }}\right)\right), t \in\left[t_{0}, \hat{t}_{f}\right]$. If $\hat{d}_{\text {min }}>0$, then the robot will most likely not collide against the object, while a collision will occur if $\hat{d}_{\min } \leq 0$.

For the braking controllers considered in this paper, the closed-loop link trajectory can be derived relatively simply. The analytical solution for the time-optimal controller is described in [13], the solutions for the other control laws are omitted for brevity. In the following, we exemplarily describe how the braking trajectory estimation of a single joint can be extended to the $n$-DOF case.

\section{A. Example}

For our analysis, we choose a double pendulum with two links weighing $m_{1}=m_{2}=4 \mathrm{~kg}$ and having length $l_{1}=l_{2}=0.5 \mathrm{~m}$. The spring stiffnesses are set to $k_{J, 1}=$ $k_{J, 2}=400 \mathrm{Nm} / \mathrm{rad}$, the maximum motor velocity is $\boldsymbol{u}_{\max }=$ $\left[\begin{array}{ll}2 & 2\end{array}\right]^{\mathrm{T}} \mathrm{rad} / \mathrm{s}$, the initial conditions are $\boldsymbol{\theta}_{0}=\left[\begin{array}{ll}0 & 0\end{array}\right]^{\mathrm{T}} \mathrm{rad}$, $\boldsymbol{q}_{0}=\left[\begin{array}{ll}0 & 0\end{array}\right]^{\mathrm{T}} \mathrm{rad}$, and $\dot{\boldsymbol{q}}_{0}=[44]^{\mathrm{T}} \mathrm{rad} / \mathrm{s}$, i.e. two times the motor velocity.

For braking the robot, we use the decoupling approach proposed in [14]. The modified controller is depicted in Fig. 9. We select the time-optimal control law for SISO braking in modal coordinates. We seek to predict how far the robot moves in the initial direction of travel and at which position it will stop. When initiating braking, we therefore determine the final and maximum link positions in the decoupled space. After transformation to original space we obtain an estimate for the maximum and final position of the entire robot. The link velocities and positions are depicted in Fig. 10 (upper left) and Fig. 10 (upper right). In the latter figure, the estimates of maximum and final link position at the very fist instant of braking are depicted, which agree well with the real values. This means that we obtain a feasible braking distance estimation for this robot. The behavior of the robot during braking as well as the braking distance estimation can also be seen in the attached video.

The braking distance prediction takes the mass and stiffness matrix into account only. A good estimation can be accomplished if the system dynamics show only a small deviation while braking. For the the results presented in Fig. 10 (top row), the eigenfrequencies vary $0.291 \%$ and $2.055 \%$ for the first and second decoupled coordinate, respectively. However, the estimation will deteriorate if non-negligible

\footnotetext{
${ }^{3}$ Of course, several obstacles and POI on the robot structure may be considered. Furthermore, other obstacle representations may be used.
} 


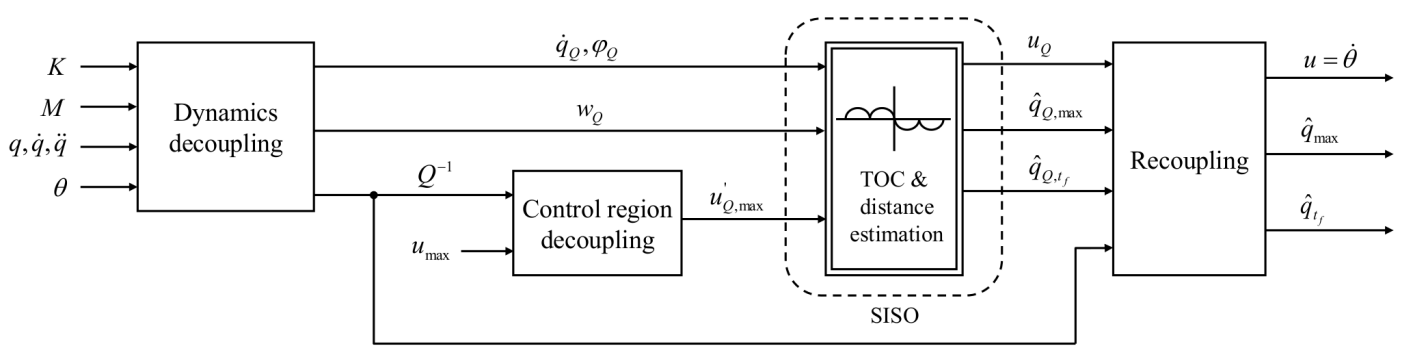

Fig. 9. Decoupling-based braking controller including braking distance estimation.
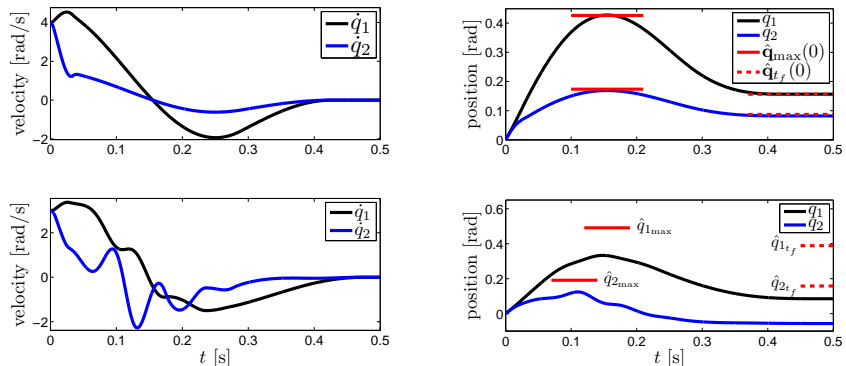

Fig. 10. Double pendulum link velocities (left) and positions (right). In the upper row the joint stiffnesses are constant, in the lower row they are nonlinear. In the right column, the estimated maximum and final position are depicted which were determined at the first instant of braking.

Coriolis torques are present and/or the mass and stiffness matrix vary significantly. This is the e.g. the case for joints with nonlinear stiffness. Figure 10 (lower row) shows results when having a nonlinear torque/deflection characteristic (21) in both joints. While braking is accomplished, we obtain a poor braking distance estimation at braking initiation. Our future research will therefore include the estimation of the timely evolution of the system dynamics in order improve the presented prediction method.

\section{CONCLUSION}

In this paper, we analyzed and compared different strategies to brake an elastic robot joint. We proposed a modification of the time-optimal control law to achieve passivity and presented two modifications of a model-free control law originally introduced in [15]. For the two model based and three model-free controllers we derived the boundary control in case of limited elastic deflection. Given the same initial conditions, we compared the performance of each controller by means of final time and passivity, and discussed extensibility to $n$-DOF elastic robots. Finally, the braking trajectories were found for estimating the braking distance of each controller. The results were applied to a double pendulum with linear joint elasticity. Given the initial state only, we were able to provide a good estimate for the maximum and terminal position. Being aware of the braking distance enables to initiate braking such that no possibly dangerous collision may occur. Our future work will consider the extension of the braking controllers and braking distance estimation to general $n$-DOF manipulators.

\section{ACKNOWLEDGMENT}

This work has been partially funded by the European Commission's Seventh Framework Programme as part of the project SAPHARI under grant no. 287513 and the project EuRoC under grant no. 608849.

\section{REFERENCES}

[1] O. Khatib, "Real-time obstacle avoidance for manipulators and mobile robots," Int. J. of Robotics Research, vol. 5, pp. 90-98, 1985.

[2] O. Brock and O. Khatib, "Elastic strips: A framework for motion generation in human environments," Int. J. Robotics Research, vol. 21, no. 12, pp. 1031-1052, 2002.

[3] E. Sisbot, L. Marin-Urias, R. Alami, and T. Simeon, "A human aware mobile robot motion planner," IEEE Transactions on Robotics, vol. 23(5), pp. 874-883, 2007.

[4] S. Haddadin, S. Haddadin, A. Khoury, T. Rokahr, S. Parusel, R. Burgkart, A. Bicchi, and A. Albu-Schäffer, "On making robots understand safety: Embedding injury knowledge into control," International Journal of Robotics, to be appeared, 2012.

[5] H. Iwata and S. Sugano, "Design of human symbiotic robot TWENDYONE," IEEE Int. Conf. on Robotics and Automation (ICRA 2009), Kobe, Japan, pp. 580-586, 2009

[6] D. Shin, I. Sardellitti, Y.-L. Park, O. Khatib, and M. Cutkosky, "Design and control of a bio-inspired human-friendly robot," The International Journal of Robotics Research, vol. 29, no. 5, pp. 571-584, apr 2010.

[7] M. Grebenstein, A. Albu-Schaffer, T. Bahls, M. Chalon, O. Eiberger, W. Friedl, R. Gruber, S. Haddadin, U. Hagn, R. Haslinger et al., "The dlr hand arm system," in IEEE International Conference on Robotics and Automation (ICRA2011), 2011. IEEE, 2011, pp. 3175-3182.

[8] S. Haddadin, F. Huber, and A. Albu-Schaffer, "Optimal control for exploiting the natural dynamics of variable stiffness robots," in IEEE International Conference on Robotics and Automation (ICRA), 2012. IEEE, 2012, pp. 3347-3354.

[9] D. J. Braun, F. Petit, F. Huber, S. Haddadin, P. van der Smagt, A. AlbuSchäffer, and S. Vijayakumar, "Robots driven by compliant actuators: optimal control under actuation constraints," 2013.

[10] M. C. Özparpucu and S. Haddadin, "Optimal control for maximizing link velocity of visco-elastic joints," in IEEE/RSJ International Conference on Intelligent Robots and Systems (IROS), 2013, pp. 30353042 .

[11] A. Bicchi and G. Tonietti, "Fast and soft arm tactics: Dealing with the safety-performance trade-off in robot arms design and control," IEEE Robotics and Automation Mag., vol. 11, pp. 22-33, 2004.

[12] S. Haddadin, A. Albu-Schäffer, O. Eiberger, and G. Hirzinger, "New insights concerning intrinsic joint elasticity for safety," in IEEE/RSJ Int. Conf. on Intelligent Robots and Systems (IROS2010), Taipeh, Taiwan, 2010, pp. 2181-2187.

[13] S. Choura and S. Jayasuriya, "An explicit minimum-time solution for the simple harmonic oscillator," Journal of Dynamic Systems, Measurement, and Control, vol. 112, no. 4, pp. 783-787, 1990.

[14] N. Mansfeld and S. Haddadin, "Reaching desired states time-optimally from equilibrium and vice versa for visco-elastic joint robots with limited elastic deflection," in IEEE/RSJ Int. Conf. on Intelligent Robots and Systems (IROS2014), 2014.

[15] F. Petit, C. Ott, and A. Albu-Schäffer, "A model-free approach to achieve damping via vibration suppression for intrinsically elastic robots," in IEEE International Conference on Robotics and Automation (ICRA), 2014, 2014.

[16] S. Wolf and G. Hirzinger, "A new variable stiffness design: Matching requirements of the next robot generation," IEEE Int. Conf. on Robotics and Automation (ICRA 2008), Pasadena, USA, pp. 17411746, 2008.

[17] E. G. Gilbert, D. W. Johnson, and S. S. Keerthi, "A fast procedure for computing the distance between complex objects in three-dimensional space," IEEE Journal of Robotics and Automation, vol. 4, no. 2, pp. 193-203, 1988. 